\title{
Tomography of the Solar Wind using Interplanetary Scintillation
}

\author{
Divya Oberoi ${ }^{1,2 *} \&$ A. Pramesh Rao, ${ }^{1 \dagger}$ \\ ${ }^{1}$ National Centre for Radio Astrophysics, Tata Institute of Fundamental Research, Pune \\ 411007. \\ ${ }^{2}$ Indian Institute of Science, Bangalore 560012. \\ *e-mail:div@ncra.tifr.res.in \\ †e-mail:.pramesh@ncra.tifr.res.in
}

Key words. Tomography-solar wind-interplanetary scintillation.

\section{Extended abstract}

Interplanetary scintillation (IPS) measurements are sensitive to a weighted sum of the properties of solar wind (SW) along the line-of-sight (los) to a distant compact radio source. Mapping a los back to the surface of the Sun provides information of the sites of origin of the SW sampled by the los. By observing different sources, lines-of-sight can be so chosen that they sample overlapping regions of Solar surface. In addition, the rotation of the Sun causes the long lived features in the SW to co-rotate, much like the twirling skirt of a ballerina, presenting different perspective views to the Earth based observers. These properties raise the possibility that systematic IPS observations can be inverted to give the maps of density and the velocity of the SW in the inner heliosphere, using techniques similar to tomography.

This technique has not been used extensively, mainly because of lack of availability of suitable datasets and the inherent complexity of the problem (Jackson et al. 1998). In order to investigate the feasibility of inverting IPS data to get distributions of velocity $(v)$ and strength of scattering $\left(C_{n}^{2}\right)$ on the solar surface, a series of simulations were undertaken. These simulations also explored the validity of the assumptions necessary for a tomographic reconstruction using IPS data. A distribution of the SW $v$ and $C_{n}^{2}$ was assumed at some fiducial surface. This distribution was propagated to fill the entire inner heliosphere accessible to IPS. The velocities were assumed to be purely radial and interaction between the fast and the slow moving SW was ignored. Lines-of-sight to radio sources were traced through the inner heliosphere and power spectra of intensity fluctuations, the primary IPS observable, constructed using the distribution of properties of the SW along the los. $v$ and $C_{n}^{2}$ were estimated for each of the lines-of-sight using a featureless SW model. A distribution of properties of SW at the fiducial surface was obtained by projecting the SW parameters estimated for all the lines-of-sight observed in one solar rotation on the fiducial surface. This distribution formed the initial guess for a $\chi^{2}$ minimisation process, the surface distribution of the SW properties formed the degrees of freedom and the power spectra along individual lines-of-sight the constraints.

The preliminary results from simulations are presented in the form of Carrington maps of $v$ and $\mathrm{C}_{n}^{2}$ for a solar rotation in Fig. 1. The features in the $\mathrm{SW}$ model, used to 


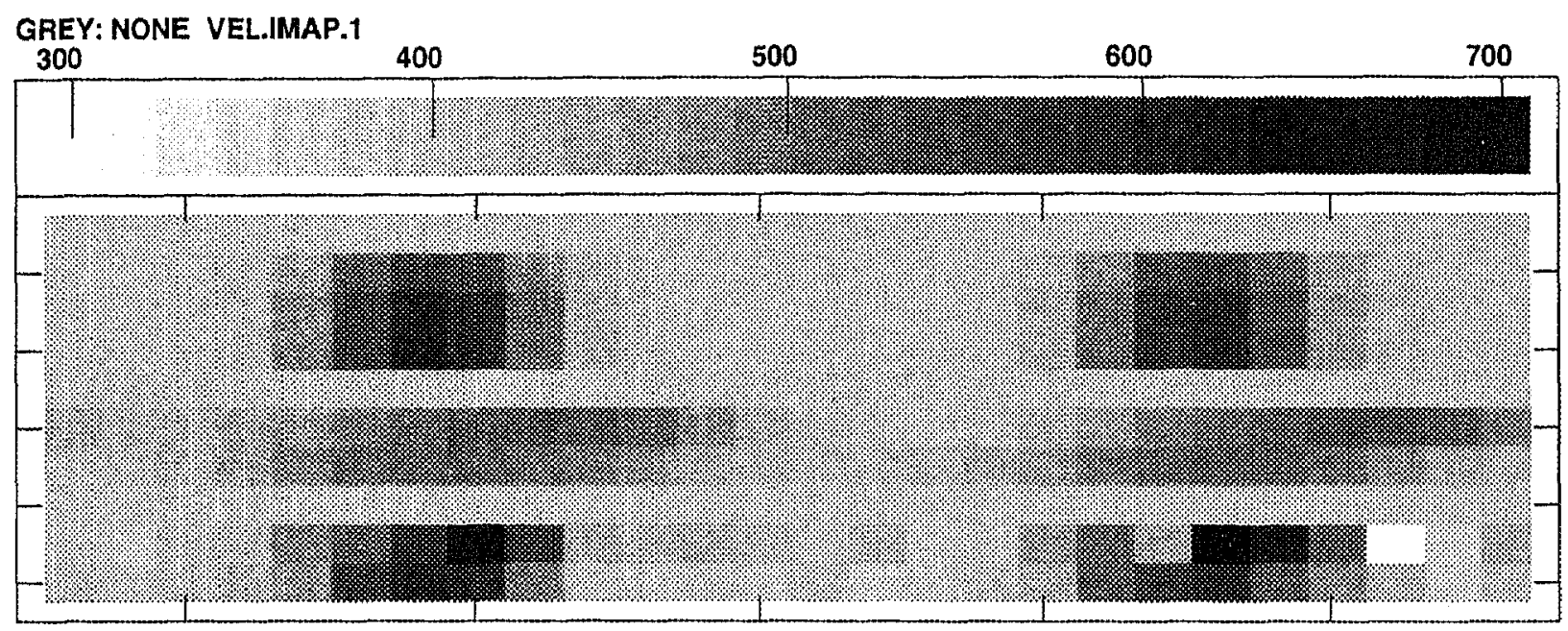

Grey scale flux range $=300.0700 .0 \mathrm{Km} / \mathrm{s}$ GREY: NONE C ${ }_{n}^{2}$.IMAP.1

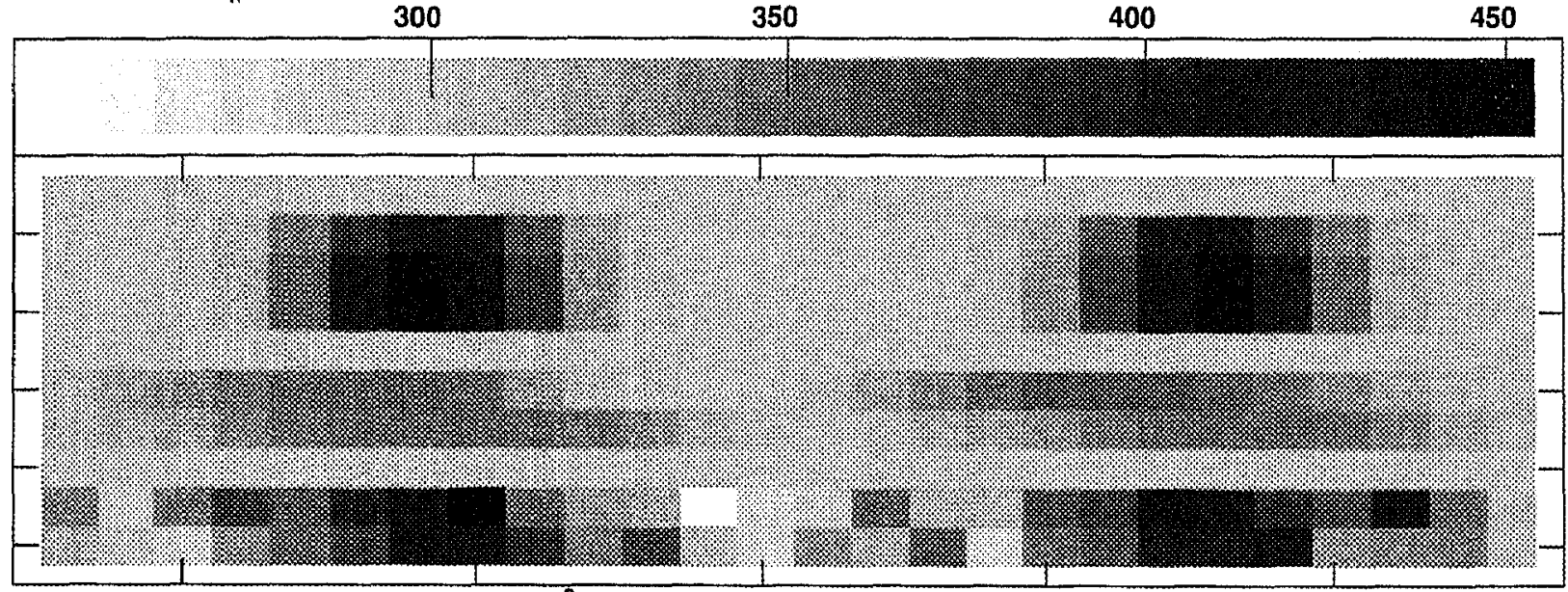

Grey scale flux range $=250.0450 .0$ Kilo $C_{n}^{2}$

Figure 1. Velocity and $C_{n}^{2}$ maps - In the top figure, the first panel shows the input model which was used to simulate the IPS data, the second panel the initial guess which was computed from the simulated data and was used as input for the reconstruction procedure and the third panel shows the final reconstructed model for velocity of the SW. The bottom figure shows the same set of plots for $C_{n}^{2}$ (in arbitrary units). The first panel shows a heliographic range of $\left[+10^{\circ},-20^{\circ}\right]$ and the second and the third panels that of $\left[+12^{\circ},-20^{\circ}\right]$. The pixel size in the reconstructed surface distribution is $13.3^{\circ}$ in longitude and $16^{\circ}$ in latitude.

generate the IPS data, were represented quite well in the reconstruction. The formal errors in the reconstruction were estimated using Monte Carlo methods and independently under the small error approximation. The two error estimates match well. The errors in $v$ range from $\sim 5 \%-13 \%$ and those in $\mathrm{C}_{n}^{2}$ from $\sim 8 \%-20 \%$.

\section{References}

Jackson, B. V. et. al. 1998, J. Geophys Res., 103, A6, 12049-12067. 